\title{
Investigation of the Efficacy and Safety of Nivolumab in Recurrent and Metastatic Nasopharyngeal Carcinoma
}

\author{
HIROKI SATO ${ }^{1}$, CHIHIRO FUSHIMI $^{2}$, TAKURO OKADA ${ }^{2}$, TAKASHI MATSUKI $^{3}$, TAKAHITO KONDO $^{4}$, \\ GO OMURA $^{5}$, KOUKI MIURA ${ }^{2}$, TAKU YAMASHITA ${ }^{3}$, ISAKU OKAMOTO ${ }^{1}$ and KIYOAKI TSUKAHARA ${ }^{1}$ \\ ${ }^{1}$ Department of Otorhinolaryngology, Head and Neck Surgery, \\ Tokyo Medical University, Tokyo, Japan; \\ ${ }^{2}$ Department of Head and Neck Oncology and Surgery, \\ International University of Health and Welfare Mita Hospital, Tokyo, Japan; \\ ${ }^{3}$ Department of Otorhinolaryngology-Head and Neck Surgery, \\ Kitasato University School of Medicine, Kanagawa, Japan; \\ ${ }^{4}$ Department of Otolaryngology, Head and Neck Surgery, \\ Tokyo Medical University Hachioji Medical Center, Tokyo, Japan; \\ ${ }^{5}$ Department of Head and Neck Surgery, National Cancer Center Hospital, Tokyo, Japan
}

\begin{abstract}
Background/Aim: Nivolumab, an anti-PD-1 inhibitor, has demonstrated efficacy in patients with several types of recurrent and metastatic $(R / M)$ squamous cell carcinoma of the head and neck. We evaluated patients with R/M-NPC receiving nivolumab. Patients and Methods: Twelve patients with R/M-NPC were enrolled at 4 institutions. The primary endpoint was overall survival, and secondary endpoints were i) progression-free survival (PFS), ii) overall response rate (ORR), iii) disease control rate $(D C R)$, and $i v)$ treatment-related toxicity. Results: The 1-year survival rate was $75.8 \%$, the median PFS was 2.8 months, and the 1-year PFS rate was $33.3 \%$. The best therapeutic response was complete response in 2, stable disease in 3 and progressive disease in 7 patients. The ORR of all patients was $16.7 \%$ and the DCR was $41.7 \%$. Conclusion: Nivolumab is a useful and relatively safe second-line systemic therapy in patients with $R / M-N P C$, and even patients who do not respond to nivolumab may survive for a long time.
\end{abstract}

This article is freely accessible online.

Correspondence to: Hiroki Sato, Department of Otorhinolaryngology, Head and Neck Surgery Tokyo Medical University, 6-7-1 Nishishinjuku, Shinjuku-ku, Tokyo 160-0023, Japan. Tel: +81 333426111, Fax: +81 333469275, e-mail: satohiro@tokyo-med.ac.jp

Key Words: Nasopharyngeal carcinoma, nivolumab, antiprogrammed death-1 monoclonal antibody, multicenter study, immune-related adverse events.
The CheckMate 141 study has demonstrated the efficacy of nivolumab, an anti-PD-1 inhibitor, in patients with recurrent and metastatic squamous cell carcinoma of the head and neck (R/M-SCCHN) (1). This study, however, has included only patients with hypopharyngeal, oropharyngeal, laryngeal, and oral carcinomas, but not those with nasopharyngeal carcinoma (NPC). Use of nivolumab in patients with recurrent and metastatic head and neck cancer, including nasopharyngeal carcinoma, who have previously received platinum-based drugs was approved in Japan in March 2017. Because PD-L1 is thought to be expressed in $89-95 \%$ of patients with NPC $(2,3)$, nivolumab, an anti-human PD-1 monoclonal antibody, was expected to be effective for the treatment of this condition. Despite this, there have been limited reports on the efficacy and safety of nivolumab in patients with NPC (4). Patients with RM-SCCHN, including laryngeal, oral, oropharyngeal, and hypopharyngeal carcinomas in the Checkmate 141 study who received nivolumab had a median survival of 7.5 months (1). In contrast, in a report by Ma et al., the median survival of patients with recurrent and metastatic NPC who received nivolumab was 17.1 months (4). Therefore, nivolumab is expected to be effective against recurrent and metastatic $\mathrm{NPC}$, however, there is no report on the actual clinical use of nivolumab in patients with recurrent and metastatic NPC. In addition, the morbidity of NPC is high in Southeast Asia (40 patients per 100,000 people annually) (5), but very low in other regions, including Japan (1 patient or fewer per 100,000 people annually) (6).

We conducted a retrospective multicenter study to investigate the efficacy and safety of nivolumab in patients with NPC in Japan. 


\section{Patients and Methods}

Patients. We retrospectively evaluated 12 patients with recurrent and metastatic NPC who started receiving nivolumab between May 1, 2017 and October 31, 2018 at 4 institutions in Japan: i) Tokyo Medical University, ii) International University of Health and Welfare Mita Hospital, iii) Kitasato University School of Medicine and iv) Tokyo Medical University Hachioji Medical Center. The observation period ended on February 28, 2019. The inclusion criteria of this study were as follows: i) patients who were histologically diagnosed with nasopharyngeal carcinoma, ii) those with distant metastasis or unresectable lesions after having received radiation therapy combined with pharmacotherapy (or without concurrent pharmacotherapy), iii) those with distant metastasis at the first visit that had received pharmacotherapy, and iv) those who had received pharmacotherapy using a regimen containing platinumbased drugs. Those who did not want to participate in the study were excluded. Patients' disease stage was determined according to the TNM classification of nasopharyngeal carcinoma, $7^{\text {th }}$ edition. Written consent was obtained from all patients for the use of their records in future studies. The study was conducted according to the Declaration of Helsinki and was approved by the ethical review board of each participating institution (T2019-0161).

Administration of nivolumab. Nivolumab was administered at $3 \mathrm{mg} / \mathrm{kg}$ (between May 1, 2017 and August 31, 2018) or $240 \mathrm{mg}$ (fixed dose) (after September 1, 2018) by intravenous drip infusion at 2-week intervals. One cycle comprised 1 dose every 2 weeks. Patients were radiographically assessed every 4-6 cycles. Treatment was continued until patients had i) clear progressive disease (PD), ii) unacceptable toxic effects occurred, or iii) until the investigators discontinued treatment for other reasons. Nivolumab was continued if the investigators judged that its administration was clinically beneficial even after observing clinical or radiographical evidence of disease progression.

Assessments. Patients were radiographically assessed every 4-6 cycles using computed tomography or magnetic resonance imaging, and treatment efficacy was determined according to the Response Evaluation Criteria in Solid Tumors (RECIST) guidelines (Version 1.1). Immune-related adverse events (irAE) that occurred due to nivolumab administration were graded according to the Common Terminology Criteria for Adverse Events (CTCAE) version 4.0.

Endpoints and statistical analysis. The primary endpoint was overall survival (OS) defined as time from treatment to death due to any cause or last follow-up. Secondary endpoints were: i) progressionfree survival (PFS), defined as interval between the initiation of treatment to first disease progression, including local recurrence, distant metastasis or death due to NPC, ii) overall response rate (ORR), iii) disease control rate (DCR), and iv) treatment-related toxicity. ORR was evaluated using the best therapeutic response. OS and PFS were determined using the Kaplan-Meier method. All statistical analyses were performed using SPSS (version 25.0, SPSS Inc., Chicago, IL, USA).

\section{Results}

Patients. The characteristics of 12 patients with recurrent and metastatic NPC who received nivolumab in this study are summarized in Table I. The patients were 30-67 years old with
Table I. Patient characteristics.

\begin{tabular}{lc}
\hline Characteristics & $\mathrm{n}(\%)$ or Median (range) \\
\hline No. of patients & 12 \\
Median age, years (range) & $58(30-67)$ \\
Gender & $10(83)$ \\
Male & $2(17)$ \\
Female & \\
ECOG PS & $11(92)$ \\
0 & $1(8)$ \\
1 & \\
Histological type & $5(41)$ \\
Keratinizing squamous cell carcinoma & $7(59)$ \\
Nonkeratinizing carcinoma & \\
EBV status & $4(33)$ \\
Positive & $2(17)$ \\
Negative & $6(50)$ \\
Unknown & \\
PD-L1 expression in tumor cells & $1(8)$ \\
Expressed $<1 \%$ & $1(8)$ \\
Expressed $\geq 1 \%$ & $8(67)$ \\
Unknown & \\
Initial clinical stage (UICC) & $2(17)$ \\
II & $3(25)$ \\
III & $3(25)$ \\
IVA & $4(33)$ \\
IVC & \\
Previous CCRT & $11(92)$ \\
Yes & $1(8)$ \\
No & \\
Site of recurrence & $5(41)$ \\
Lung & $3(25)$ \\
Liver & $3(25)$ \\
Lone & $8(67)$ \\
Nasopharynx & $2(17)$ \\
No. of prior lines of chemotherapy & \\
1 & $2(17)$ \\
2 & $5(41)$ \\
3 & $3(25)$ \\
$\geq 4$ & $2(17)$ \\
\hline
\end{tabular}

ECOG PS: Eastern Cooperative Group performance status; EBV: EpsteinBarr virus; PD-L1: programmed death-ligand 1; CCRT: concurrent chemoradiotherapy.

a median age of 58 years. There were 10 males and 2 females. At the first visit, 2 patients had UICC Stage II, 3 patients had UICC Stage III, 3 patients had UICC Stage IVA, and 4 patients had UICC Stage IVC. Eleven of the 12 patients received concurrent chemoradiotherapy, which included i) cisplatin monotherapy in 6 patients; ii) combination therapy with cisplatin, 5FU, and docetaxel in 2 patients; iii) combination therapy with nedaplatin and S-1 in 2 patients; and iv) combination therapy with cisplatin and 5FU in 1 patient. Regarding the site of recurrence, i) lung metastases were observed in 5 patients, ii) liver metastases in 3 patients, iii) bone metastases in 3 patients, and iv) lymph nodes metastases 


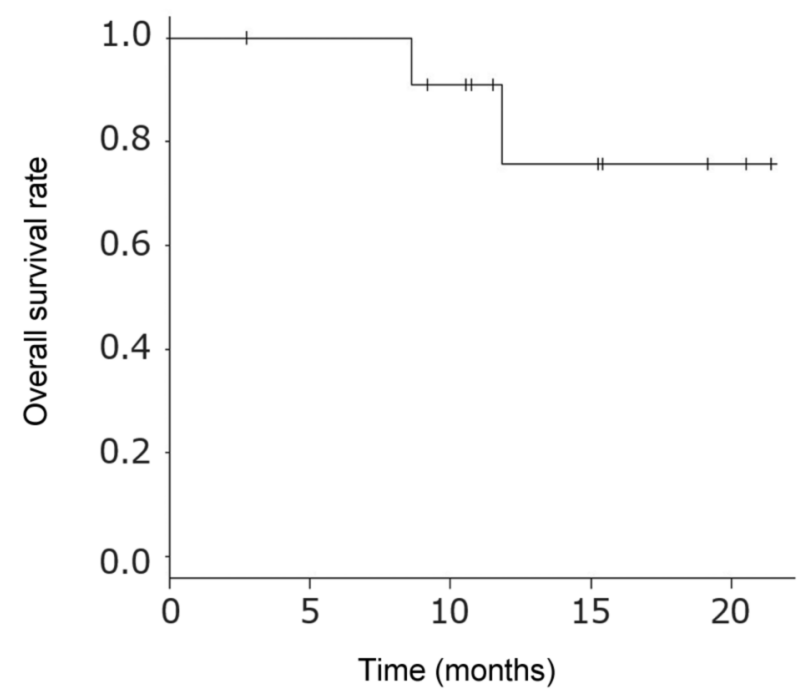

Figure 1. Overall survival. The 1-year survival rate was $75.8 \%(95 \%$ confidence interval=30.5-93.7\%).

in 8 patients (cervical lymph nodes in 4 patients, mediastinal lymph nodes in 2 patients, and abdominal lymph nodes in 2 patients). Two patients had local recurrence in the nasopharynx. The initial treatment regimen for recurrence and metastasis was i) nivolumab in 4 patients, ii) paclitaxel+cetuximab in 2 patients, iii) $\mathrm{S}-1$ in 2 patients, cisplatin+5FU+cetuximab in 1 patient, iv) carboplatin $+5 \mathrm{FU}$ in 1 patient, v) Docetaxel, cisplatin and fluorouracil (TPF) therapy in 1 patient, and vi) gemcitabine in 1 patient. Three patients received nivolumab as second-line therapy.

Efficacy. The median duration of observation for all patients was 11.9 months (range=2.8-21.7 months). Nine patients survived, 2 patients died of the primary disease, and 1 patient was lost to follow-up. The 1-year survival rate of all patients was $75.8 \%$ [ $95 \%$ confidence interval $(\mathrm{CI})=30.5-93.7 \%$ ] (Figure 1). The median PFS of all patients was 2.8 months $(95 \% \mathrm{CI}=0.5-$ 5.0), and the 1-year PFS rate was $33.3 \%$ (Figure 2).

The best therapeutic response was complete response (CR) in 2 patients $(17 \%)$, stable disease in 3 patients and progressive disease in 7 patients (Figure 3). None of the patients had partial response as a best therapeutic response. One patient achieved PR due to treatment with paclitaxel and cetuximab received following treatment with nivolumab (Figures 4 and 5). Thus, the ORR of all patients was $16.7 \%$ and the DCR was $41.7 \%$.

Immune-related adverse events. Adverse events experienced by the 12 patients who received nivolumab are shown in Table II. Six patients experienced adverse events of any grade, with liver dysfunction occurring in 2 patients, interstitial lung disease in 2 patients, anemia in 1 patient,

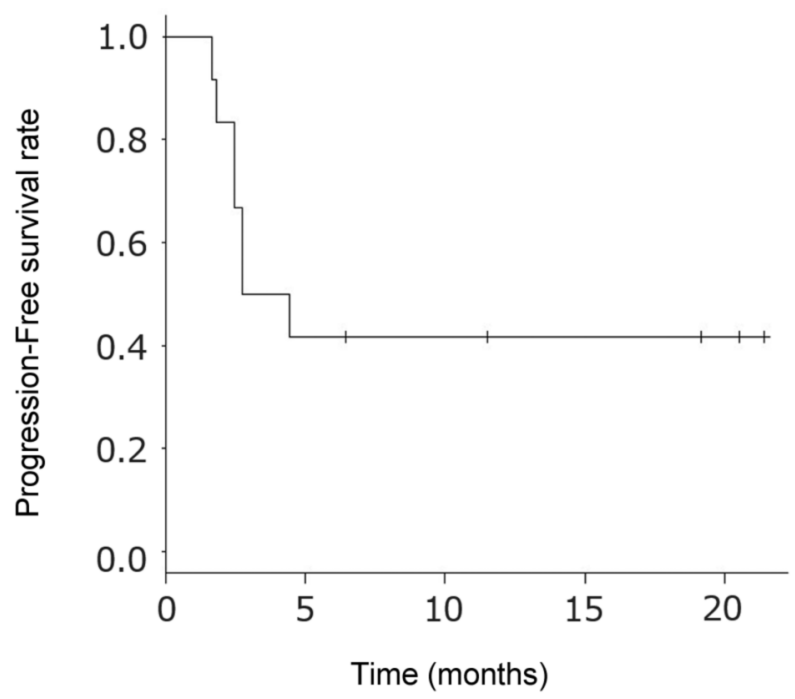

Figure 2. Progression-free survival. Median progression-free survival was 3.6 months (95\% confidence interval=1.8 months-NA). The 1-year progression-free survival rate was $41.7 \%$ (95\% confidence interval=15.2$66.5 \%)$.

hypothyroidism in 1 patient, arthritis in 1 patient, dermatitis in 1 patient, and myositis in 1 patient. Liver dysfunction observed in 1 patient was the only irAE of Grade 3 or higher. Administration of nivolumab was stopped following the development of liver dysfunction.

\section{Discussion}

Recurrent and metastatic nasopharyngeal carcinoma has poor prognosis and is typically treated with combination therapy with fluorouracil and cisplatin (FP). Recently, however, a multicenter randomized clinical study in China, which verified the efficacy of combination therapy with gemcitabine and cisplatin (GP) compared to FP, reported that the PFS, the primary endpoint of the study, was significantly better in the GP group than in the FP group $[\mathrm{PFS}=7$ months, 95\% CI=6.3-7.6 vs. 5.6 months, $95 \% \mathrm{CI}=4.9-6.2$; hazard ratio $(\mathrm{HR})=0.55,95 \% \mathrm{CI}=0.44-0.68$, $p<0.0007]$ (7). In the same study, the median survival time was also significantly better in the GP group than in the FP group (MST=29.1 months, 95\% CI=18.7-35.9 vs. 20.9 months, $95 \% \mathrm{CI}=16.0-25.8 ; \mathrm{HR}=0.62,95 \% \mathrm{CI}=0.45-0.84, p=0.0025)$. As a result of this phase III study, combination therapy with gemcitabine+cisplatin is becoming a standard of care for recurrent and metastatic NPC worldwide. However, because the use of gemcitabine for nasopharyngeal carcinoma is not approved in Japan, the GP regimen cannot be used. Instead, FP is usually used to treat recurrent and metastatic NPC. In cases where a patient with recurrent and metastatic nasopharyngeal carcinoma does not respond to treatment with FP, other treatment options are currently very limited in Japan. 


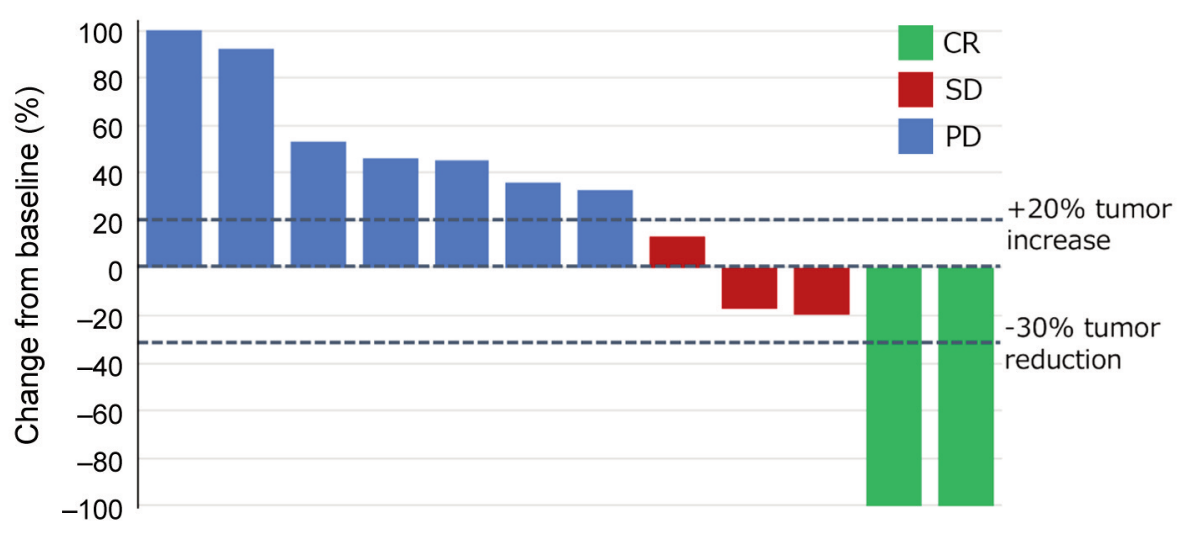

Figure 3. Waterfall plot showing the best therapeutic response. Complete response was seen in 2 patients (16.7\%), stable disease in 3 patients (25\%) and progressive disease in 7 patients (58.3\%). The response rate was $16.7 \%$ and the disease control rate was $41.7 \%$. CR: Complete response; SD: stable disease; $P D$ : progressive disease.

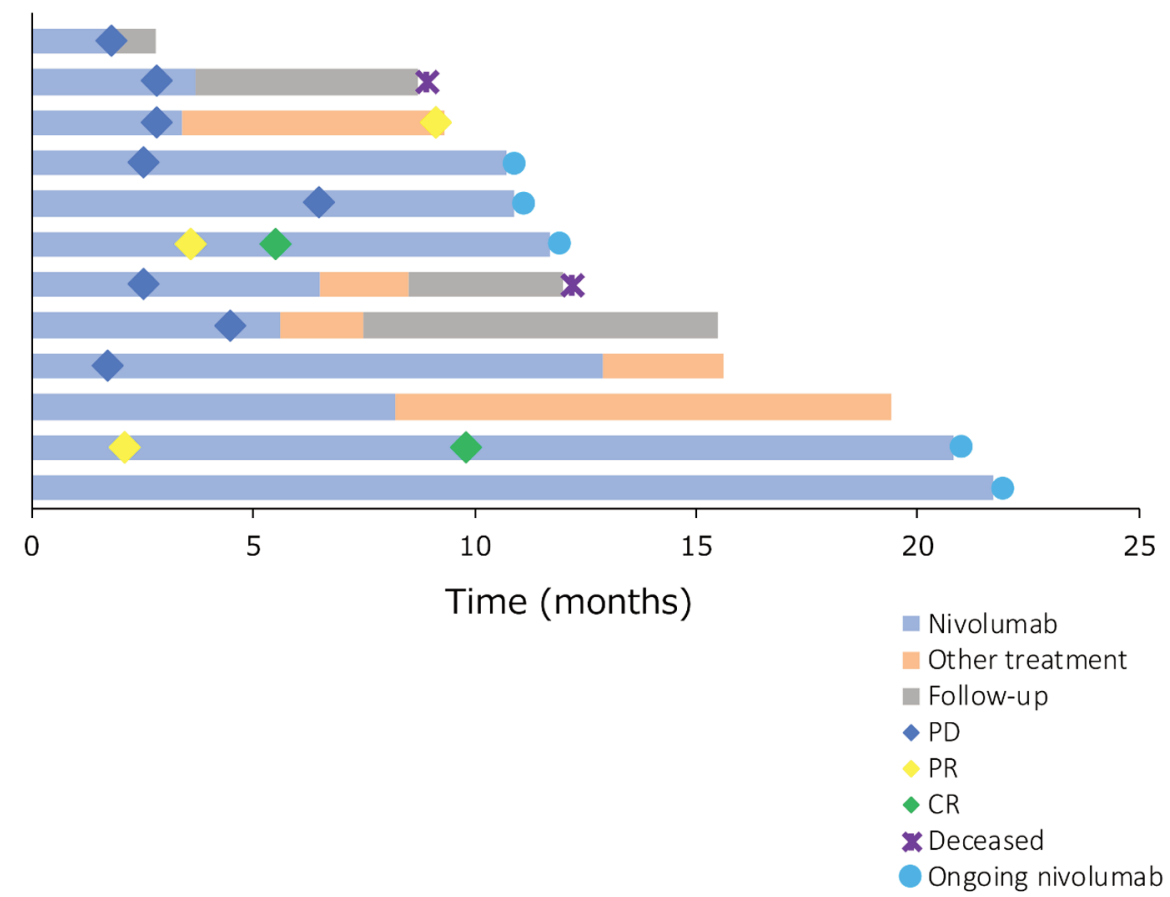

Figure 4. Swimmer plot showing nivolumab treatment was ongoing in all responders. Long-term survivors were not necessarily responders. CR: Complete response; PR: partial response; PD: progressive disease.

The efficacy of the immune checkpoint inhibitors, nivolumab and pembrolizumab, has been demonstrated against recurrent and metastatic nasopharyngeal carcinoma in recent years $(4,8)$, and immunotherapy is described as a subsequent-line therapy in the NCCN guidelines. In a study on pembrolizumab by Hsu et al. in 27 patients with recurrent and metastatic nasopharyngeal carcinoma in whom PD-L1 was expressed in at least $1 \%$ of tumor cells or tumor infiltrating lymphocytes, the median survival time was 16.5 months $(95 \% \mathrm{CI}=10.1-$ not reached), the 1 -year survival rate was $63.0 \%$, the median recurrence-free survival time was 6.5 months (95\% CI=3.6-13.4), and the 1-year PFS rate was $33.4 \%$. Moreover, the ORR was $25.9 \%$ and the DCR was $77.8 \%$ (8). In a study on nivolumab by Ma et al. in 44 patients with recurrent and metastatic nasopharyngeal carcinoma (irrespective of the presence/absence of PD-L1 expression), the median survival time was 17.1 months (95\% CI $=10.9$-not reached), the 1-year survival rate was $59 \%$ $(95 \% \mathrm{CI}=44.3-78.5 \%)$, the median recurrence-free survival time was 2.8 months (95\% CI=1.8-7.4), and the 1 -year PFS 


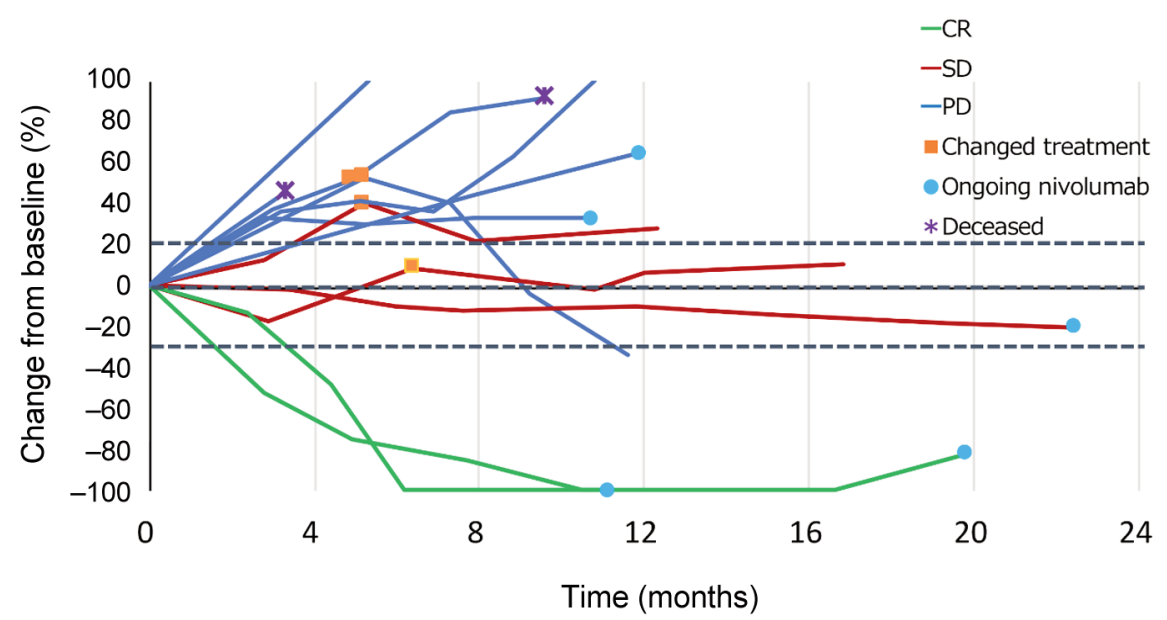

Figure 5. Spider plot. This shows some long-term survivors had tumors that did not grow but instead remained unchanged after maintaining SD or PD for a long time. CR: Complete response; SD: stable disease; PD: progressive disease.

rate was $19.3 \%(95 \% \mathrm{CI}=10.1-37.2 \%)$. Moreover, the ORR was $20.5 \%$ and the DCR was $54.5 \%$ (4). In the current study, 9 of the 12 patients survived with a 1-year survival rate of $75.8 \% \quad(95 \% \mathrm{CI}=30.5-93.7 \%)$, median recurrence-free survival time of 3.6 months (95\% CI=1.8 months-NA), 1year PFS rate of $41.7 \%(95 \% \mathrm{CI}=15.2-66.5 \%)$, ORR of $16.7 \%$, and DCR of $41.7 \%$. The ORR and DCR in the present study are slightly lower, while the 1-year survival rate and PFS are comparable to those reported the previous studies. Based on a swimmer plot of findings from the present study, possible reasons why the survival rate was equal to that reported previously despite the lower ORR and DCR are: i) some of the patients continued to receive nivolumab for a relatively long period of time after the therapeutic effect was judged to be PD, and ii) some of the patients continued to receive subsequent treatment following the nivolumab treatment for a relatively long period of time. Moreover, a spider plot of findings from the present study indicates that the presence of patients who i) maintained stable disease (SD) for a long time, ii) survived for a long time after subsequent treatment following nivolumab treatment, or iii) continued to receive nivolumab for a relatively long period of time despite the therapeutic effect having been judged to be PD, contributed to their extended survival. A study on long-term (5 years or longer) outcomes of patients with non-small-cell lung carcinoma, which differs from nasopharyngeal carcinoma, has reported several longterm (5 years or longer) survivors following nivolumab treatment. These patients included those whose best therapeutic response was either SD or PD (9). Thus, as with patients with non-small-cell lung carcinoma, patients with recurrent and metastatic nasopharyngeal carcinoma may survive for a long time even if their best therapeutic response after nivolumab treatment is SD or PD.
Table II. Immune-related adverse events. Values indicate number of patients, from a total of 12 patients. Treatment was discontinued in the patient with Grade 3 liver dysfunction.

\begin{tabular}{lcccc}
\hline Adverse event & Any grade & Grade 1 & Grade 2 & Grade 3 \\
\hline Liver dysfunction & 2 & & 1 & 1 \\
Interstitial lung & 2 & 1 & 1 & \\
disease & 1 & & 1 & \\
Anemia & 1 & 1 & & \\
Hypothyroidism & 1 & & & \\
Arthritis & 1 & 1 & & \\
Dermatitis & 1 & 1 & 3 & 1 \\
Myositis & 9 & 4 & 3 & \\
Total & & & & \\
\hline
\end{tabular}

Based on studies performed previously, at present, combination therapy with cisplatin and gemcitabine should be selected as the first-line systemic therapy for recurrent and metastatic nasopharyngeal carcinoma. In Japan, where gemcitabine cannot be used, FP therapy may be useful. Immunotherapy, including nivolumab, is considered a very useful second-line systemic therapy for recurrent and metastatic nasopharyngeal carcinoma. This is because 1 -year survival rates of patients treated with regimens comprising single agents, such as platinum-based drugs and taxanes, which are recommended by the NCCN guidelines, are all poor ( $40 \%$ or lower). Only gemcitabine monotherapy is a suitable second-line therapy, with the 1-year survival rate of patients at $67 \%$, which is comparable to that of immunotherapy.

Adverse events occurred in 6 of the 12 patients $(50 \%)$ in the present study. Apart from 1 case of Grade 3 liver dysfunction all adverse events were Grade 2 or lower. In contrast, the report by Ma et al. found that irAE of Grade 3 or higher, including 
Grade 5, due to nivolumab administration occurred in $22 \%$ of patients with recurrent and metastatic nasopharyngeal carcinoma (4). A report by Hsu et al. found that irAE of any grade due to pembrolizumab administration occurred in $74.1 \%$ of patients with recurrent and metastatic nasopharyngeal carcinoma, and irAE of Grade 3 or higher, including Grade 5, occurred in $55.6 \%$ of patients (6). These incidences are higher than those of adverse events due to nivolumab in patients with squamous-cell carcinoma of the head and neck, not including nasopharyngeal carcinoma (adverse events of any grade, $58.9 \%$; adverse events of Grade 3 or higher, $13.1 \%$ ). Therefore, administration of immunotherapy to patients with recurrent and metastatic nasopharyngeal carcinoma in actual clinical practice should be accompanied by sufficient attention to the occurrence of irAE, including death.

Limitations of the present study include its retrospective nature and the small sample size (12 patients). The small number of enrolled patients may have artificially skewed the overall survival rate and the occurrence of adverse events to lower values. However, the present study provides useful information, suggesting that long-term survival may be possible for patients with recurrent and metastatic nasopharyngeal carcinoma, not only for those who respond to treatment with nivolumab but also those with a therapeutic effect of SD or PD in actual clinical practice.

Nivolumab is a useful second-line systemic therapy for patients with recurrent and metastatic nasopharyngeal carcinoma. Additionally, even patients who do not respond to nivolumab may survive for a long time. Nivolumab is also relatively safe in terms of irAE, however, sufficient attention should be paid to the occurrence of irAE, including death.

\section{Conflicts of Interest}

K. Tsukahara has received grant support from Ono. Other Authors have no conflicts of interest, potential conflicts or financial relationships to disclose.

\section{Authors' Contributions}

HS and IO designed the study. HS wrote the manuscript text and prepared the figures. HS, CF, TO, TM, TK and IO collected the data. HS, IO, and GO involved in analysis and interpretation of data. KT offered scientific advice. KM, TY and KT were the supervisors. All Authors reviewed and approved the final article.

\section{References}

1 Ferris RL, Blumenschein G, Jr., Fayette J, Guigay J, Colevas $\mathrm{AD}$, Licitra L, Harrington K, Kasper S, Vokes EE, Even C, Worden F, Saba NF, Iglesias Docampo LC, Haddad R, Rordorf T, Kiyota N, Tahara M, Monga M, Lynch M, Geese WJ, Kopit J, Shaw JW and Gillison ML: Nivolumab for recurrent squamous-cell carcinoma of the head and neck. N Engl J Med 375(19): 1856-1867, 2016. DOI: 10.1056/NEJMoa1602252
2 Fang W, Zhang J, Hong S, Zhan J, Chen N, Qin T, Tang Y, Zhang Y, Kang S, Zhou T, Wu X, Liang W, Hu Z, Ma Y, Zhao Y, Tian Y, Yang Y, Xue C, Yan Y, Hou X, Huang P, Huang Y, Zhao $\mathrm{H}$ and Zhang L: Ebv-driven Imp1 and ifn-gamma upregulate pd-11 in nasopharyngeal carcinoma: Implications for oncotargeted therapy. Oncotarget 5(23): 12189-12202, 2014. PMID: 25361008. DOI: 10.18632/oncotarget.2608

3 Zhang J, Fang W, Qin T, Yang Y, Hong S, Liang W, Ma Y, Zhao $\mathrm{H}$, Huang Y, Xue C, Huang P, Hu Z, Zhao Y and Zhang L: Coexpression of pd-1 and pd-11 predicts poor outcome in nasopharyngeal carcinoma. Med Oncol 32(3): 86, 2015. PMID: 25702326. DOI: $10.1007 / \mathrm{s} 12032-015-0501-6$

4 Ma BBY, Lim WT, Goh BC, Hui EP, Lo KW, Pettinger A, Foster NR, Riess JW, Agulnik M, Chang AYC, Chopra A, Kish JA, Chung CH, Adkins DR, Cullen KJ, Gitlitz BJ, Lim DW, To KF, Chan KCA, Lo YMD, King AD, Erlichman C, Yin J, Costello BA and Chan ATC: Antitumor activity of nivolumab in recurrent and metastatic nasopharyngeal carcinoma: An international, multicenter study of the mayo clinic phase 2 consortium (nci9742). J Clin Oncol 36(14): 1412-1418, 2018. PMID: 29584545. DOI: $10.1200 / J C O .2017 .77 .0388$

5 Cao SM, Simons MJ and Qian CN: The prevalence and prevention of nasopharyngeal carcinoma in china. Chin J Cancer 30(2): 114-119, 2011. PMID: 21272443. DOI: 10.5732/ cjc. 010.10377

$6 \mathrm{Yu} \mathrm{MC}$ and Yuan JM: Epidemiology of nasopharyngeal carcinoma. Semin Cancer Biol 12(6): 421-429, 2002. PMID: 12450728. DOI: 10.1016/s1044579x02000858

7 Zhang L, Huang Y, Hong S, Yang Y, Yu G, Jia J, Peng P, Wu X, Lin Q, Xi X, Peng J, Xu M, Chen D, Lu X, Wang R, Cao X, Chen X, Lin Z, Xiong J, Lin Q, Xie C, Li Z, Pan J, Li J, Wu S, Lian Y, Yang Q and Zhao C: Gemcitabine plus cisplatin versus fluorouracil plus cisplatin in recurrent or metastatic nasopharyngeal carcinoma: A multicentre, randomised, openlabel, phase 3 trial. Lancet 388(10054): 1883-1892, 2016. PMID: 27567279. DOI: 10.1016/S0140-6736(16)31388-5

8 Hsu C, Lee SH, Ejadi S, Even C, Cohen RB, Le Tourneau C, Mehnert JM, Algazi A, van Brummelen EMJ, Saraf S, Thanigaimani P, Cheng JD and Hansen AR: Safety and antitumor activity of pembrolizumab in patients with programmed death-ligand 1-positive nasopharyngeal carcinoma: Results of the keynote-028 study. J Clin Oncol 35(36): 40504056, 2017. PMID: 28837405. DOI: 10.1200/JCO.2017.73.3675

9 Gettinger S, Horn L, Jackman D, Spigel D, Antonia S, Hellmann M, Powderly J, Heist R, Sequist LV, Smith DC, Leming P, Geese WJ, Yoon D, Li A and Brahmer J: Five-year follow-up of nivolumab in previously treated advanced non-small-cell lung cancer: Results from the ca209-003 study. J Clin Oncol 36(17): 1675-1684, 2018. PMID: 29570421. DOI: $10.1200 / \mathrm{JCO}$ 2017.77.0412

Received June 9, 2020

Revised July 7, 2020

Accepted July 10, 2020 